\title{
Traditional and Mobile Public Health Alert Communications with Health Care Providers
}

\author{
Debra Revere*, lan Painter and Janet Baseman \\ University of Washington, Seattle, WA, USA
}

\section{Objective}

To systematically compare mobile (SMS) and traditional (email, FAX) communication strategies to identify which modality is most effective for communication of health alerts and advisories between public health agencies and health care providers in order to improve emergency preparedness and response.

\section{Introduction}

The effectiveness of emergency preparedness and response systems depends, in part, on the effectiveness of communication between agencies and individuals involved in emergency response, including health care providers who play a significant role in planning, event detection, response and communication with the public. Although much attention has been paid to the importance of communicating clinical data from health care providers to public health agencies for purposes of early event detection and situational awareness (e.g., BioSense) and to the need for alerting health care providers of public health events (e.g., Health Alert Networks), no studies to date have systematically identified the most effective methods of communication between public health agencies and community health care providers for purposes of public health emergency preparedness and response.

The REACH (Rapid Emergency Alert Communication in Health) study is a 4-year randomized controlled trial to evaluate and compare the effectiveness of mobile (SMS) and traditional (email, FAX) communication strategies for sending public health messages to health care providers - physicians, pharmacists, nurse practitioners, physician's assistants and veterinarians.

\section{Methods}

Providers were recruited from three sites (King County, WA; Spokane County, WA; and across the state of Montana; N=845) and randomized to receive time-sensitive public health messages via email, Fax, short message service (SMS) or to a control group that did not receive messages. For one year, alerts based on real events of public health interest were sent quarterly with follow-up telephone interviews conducted 5-10 days after the delivery date. Interviews consisted of approximately six questions that elicited information about message receipt, recall of its content and perceived credibility and trustworthiness of the message and source. In addition, provider access to online alert information and delivery success or failure was collected.

\section{Results}

Frequency of receipt, timeliness, content awareness, perceived credibility and perceived trustworthiness were measured and com- pared across communication delivery systems. On average $84.0 \%$ of participants were contacted in each follow-up survey following all alerts and across all three sites. Primary data analysis was designed to measure differences between the three communication groups using intent-to-treat methods. A set of secondary analyses examined the outcomes excluding providers who could not have received messages (due to incorrect contact information, known technical failures, or because providers could not receive messages by the assigned delivery message - for example, a provider without a Fax number randomized to the Fax group).

We will discuss preliminary results of intent-to-treat analyses regarding rate of recall of study alert message content between traditional and mobile communications and perceived trustworthiness and credibility of message and message source by providers. In addition, we will report on frequency of accessing online alert information between traditional and mobile delivery groups.

\section{Conclusions}

There is currently no evidence-based research to guide or improve the practice of public health communication between public health agencies and health care providers before, during and after a public health emergency. Improving this communication via the use of effective media can enhance disease surveillance, which will aid in early detection and enhance case finding and situational awareness for public health emergencies. By systematically evaluating the relative effectiveness of mobile and traditional message delivery systems for emergency preparedness and response communications, the REACH study contributes to building the evidence base for novel and effective approaches to emergency communications.

\section{Keywords}

Emergency Preparedness and Response; Public Health Communication; Surveillance and Alerting

\section{Acknowledgments}

This work was made possible by the CDC Office of Public Health Preparedness and Response Extramural Research Program Preparedness and Emergency Response Research Centers award, Grant no. 5P01TP000297, to the University of Washington. The contents are solely the responsibility of the authors and do not necessarily represent the official views of the CDC.

\section{*Debra Revere}

E-mail: drevere@uw.edu 